\title{
Clearance of technetium-99m-DTPA in pulmonary sarcoidosis
}

\author{
K.M. Antoniou1, N. Tzanakis1, K. Malagari2, K.E. Symvoulakis1, \\ K. Perisinakis 3 , N. Karkavitsas, N.M. Siafakas ${ }^{1}$, D.E. Bouros ${ }^{4}$
}

ABSTRACT: Clearance of technetium-99m-DTPA in pulmonary sarcoidosis. K.M. Antoniou, N. Tzanakis, K. Malagari, K.E. Symvoulakis, K. Perisinakis, N. Karkavitsas, N.M. Siafakas, D.E. Bouros.

Background. The aim of this study was to explore the possible association of the lung clearance of $99 \mathrm{mTc}$ DTPA scan with HRCT lung abnormalities and with the pulmonary function tests [PFTs] in patients with sarcoidosis.

Methods. We studied prospectively 15 patients [5 males, 10 females] of median age 46yr [range 27-67] with histologically proved sarcoidosis. HRCT scoring included the sum of the severity and extent of lymph node enlargement and parenchymal involvement.
Results. The mean DTPA clearance half-time $[\tau \mathbf{1} / 2$ $<40 \mathrm{~min}$ ] was found [mean [SD]] $38.3 \pm 4.5 \mathrm{~min}$. The lymph node enlargement was found $34 \%$ and the parenchymal involvement $12 \%$. DTPA clearance was negatively correlated with the parenchymal involvement $[r=-0.651$, $p=0.009]$. The HRCT parenchymal abnormalities were found significantly correlated with PFTs $[\mathrm{FVC}[\mathrm{r}=\mathbf{- 0 . 6 5}$, $p=0.008]$ and TLCO $[r=-0.76, p=0.02]$.

Conclusions. Our data suggest a moderate association between ${ }^{99 m}$ Tc-DTPA scan and HRCT in pulmonary sarcoidosis. However, further studies in large scale of sarcoid patients are needed to clarify the role of this novel methodology in the evaluation and follow-up of this disorder. Monaldi Arch Chest Dis 2006; 65: 3, 141-144.

Keywords: Sarcoidosis, DTPA clearance, HRCT, radionuclear scan.

1 Thoracic Medicine, Medical School University of Crete, Heraklion,

2 Radiology, Medical School University of Athens,

3 Nuclear Medicine, Medical School University of Crete, Heraklion,

4 Pneumonology,Democritus University of Thrace, Alexandroupolis, Greece.

Correspondence: Demosthenes Bouros; Professor of Pneumonology; Democritus University of Thrace, 68100 Alexandroupolis, Thrace,Greece; e-mail: bouros@med.duth.gr

\section{Introduction}

Inflammation of lower airways characterizes the lung injury and fibrosis In interstitial lung disease [ILD]. Clearance of inhaled technetium-labeled diethylenetriamine pentaacetate [99mTc-DT$\mathrm{PA}]$ from the lung is an index of lung epithelial permeability [1]. Increased DTPA clearance may be a sensitive marker of inflammation [2] and normal clearance certifies absence of inflammation [3]. It is abnormal in several ILD, it has been used as a marker of alveolar inflammation in sarcoidosis $[3,4]$ and could potentially be used as a predictor of disease progression in Idiopathic Pulmonary Fibrosis (IPF) [5]. On the contrary, recent data suggest that 99mTc-DTPA is weakly associated with high-resolution computed tomography (HRCT) in IPF, while has been found abnormal in the majority of the evaluated patients [6]. The technique is non invasive, relatively inexpensive with a low radiation, and easy to perform using standard equipment in clinical nuclear medicine departments $[7,8]$.

HRCT has diagnostic and prognostic value, and should be a part of the initial evaluation of ILD [9]. For most sarcoidosis patients, HRCT scans are indicated [10].
The objective of this study was to explore the degree of association of HRCT score of pulmonary and lymph node involvement and the rate of clearance of $99 \mathrm{mTc}$-DTPA in sarcoidosis patients. Furthermore, we investigated the correlation between the rate of DTPA clearance and certain indices of pulmonary function tests [PFTs].

\section{Methods}

\section{Patients}

Fifteen sarcoidosis patients [5 males, $10 \mathrm{fe}$ males] of median age 46yr [range 27-67] with histologically proven sarcoidosis, who were investigated in the ILD clinic of the University Hospital of Heraklion were enrolled in the study. The ATS/ERS/WASOG statement [10] on sarcoidosis was followed for the diagnosis, based on history, clinical symptoms, standard chest radiography, HRCT scan, lung Ga67 scintigraphy and laboratory tests [serum angiotensin-converting enzyme]. All of them had transbronchial or open lung biopsy with histopathological evidence of noncaseating epithelioid cell granulomas.

Patients were excluded from the study if they were current smokers during the 6 months prior to 
the measurement of DTPA clearance. According to chest radiography classification of sarcoidosis, 5 had type I disease, 7 type II, and 3 type III disease [10].

\section{Methods}

Spirometry was performed with a computerized system [MasterLab, Jaeger2.12, Germany]. The measurement was performed using standard protocols according to American Thoracic Society (ATS) guidelines [11]. Subjects did not use short acting bronchodilators within 12 hours prior to the measurements. The best of three consecutive measurements was chosen.

\section{HRCT in sarcoidosis patients}

CT scoring included the sum of the severity and extent of lymph node enlargement at three different sites (paratracheal, mediastinal/carina, hilar) and the parenchymal involvement [peribronchial thickening, nodules, collapse/consolidation/pseudoalveolar opacities, ground glass, and reticulation/honeycombing].

For lymph node involvement, mediastinal windows and levels were used and a semi-quantitative grading was done ranging from $0-1$ for each of the above lymph node sites as follows: No lymph node enlargement was graded as 0 ; lymph nodes with a diameter $<10 \mathrm{~mm}$ as 1 , and nodes $>10 \mathrm{~mm}$ as 2 . Therefore for a patient with all the above lymph sites involved the maximum score would be 6 .

For parenchymal evaluation HRCT images were used and were subsequently evaluated at five different levels recording the relative proportion of all the above findings to within 5\%. Scores from the five HRCT slices were summed and divided by the total number of slices to calculate the average extent score for each of the variables. The levels of evaluation included the vascular pedicle, the tracheal carina, the pulmonary hilum, the pulmonary venous confluence, and 1-2 $\mathrm{cm}$ above the right diaphragm. The scans were performed with $1 \mathrm{~mm}$ thickness and a 1 to $2 \mathrm{~s}$ scanning time during breath holding at end inspiration. High spatial frequency algorithm was used and images were read at window levels appropriate for pulmonary parenchyma (level -600 to 700 Hounsfield units: width 1600 Hounsfield units). For evaluation of extent of parenchymal involvement was assessed by HRCT slices using a visual method, similar to that used previously by other investigators $[12,13]$. For each of the parenchymal features evaluated: 1 . peribronchial thickening, 2. nodules, 3. collapse/consolidation/pseudoalveolar opacities, 4. ground glass, and 5. reticulation/honeycombing] quantification was done as follows: no abnormality graded as 0 ; extent $<25 \%$ as 1 ; extent $25-50 \%$ as 2 ; extent $>50 \%$ as 3 . Therefore, for each patient the range of lung parenchyma score would be $0-15$. The maximum sum of involvement for each patient is $21(15+6)$. This scoring system is similar to the Oberstein et al. scoring with a slight modification [in our study we did not evaluate pleural changes as in previous studies were not correlated with any disease activity index] [12].

\section{9mTc-DTPA scan}

An aerosol of Tc-99m diethylenetriamine pentaacetate [DTPA] was produced using a Venticis II radioaerosol delivery system [CIS bio international, Cedex, France]. The radioaerosol was administered to patients for 4 minutes. A dynamic study consisting of 30 one-minute frames were then acquired by a GE Millennium MPS $\gamma$-camera [GE, Milwuakee, Wisconsin, USA].

Regions of interest [ROI] were identified on either lung and a background ROI was set. Background subtraction was employed and an exponential fit of the clearance of each lung was derived starting from the frame with peak activity. The halftime of clearance was estimated in minutes for each lung and the average value was obtained. A clearance half-time of $<40 \mathrm{~min}$ was regarded as abnormal and a clearance half-time of less than $20 \mathrm{~min}$ [50\% of normal] was categorized as very rapid $[5,14,15]$.

\section{Results}

The demographic and clinical characteristics of the sarcoidosis patients are shown in table 1. The mean $($ mean \pm SD) DTPA clearance half-time was found $38.3 \pm 4.5 \mathrm{~min}$, (range: $31-45$ ). The scans were evaluated as abnormal in 10 of 15 of our patients $(66.7 \%)$. For lymph node involvement, the scoring system results were $2.07 \pm 1.22$ (range 0 to 4 ) and with a $34 \%$ in $\%$ evaluation. For parenchymal involvement the scoring system results were $2.53 \pm 3.48$ (range 0 to 10) and with a $12 \%$ in a $\%$ evaluation. DTPA clearance rate was significantly correlated with HRCT scoring of parenchymal involvement $(\mathrm{r}=$ $-0.651, \mathrm{p}=0.009$ ) (figure 1). The HRCT parenchymal abnormalities were found significantly correlated with PFTs [FVC $(r=-0.65, \mathrm{p}=0.008)$ and TLCO $(\mathrm{r}=-$ $0.76, \mathrm{p}=0.02)]$. No correlation was found between parenchymal abnormalities and $\mathrm{FEV}_{1}(\mathrm{r}=-0.15$, $\mathrm{p}=0.9)$ such as with TLC $(\mathrm{r}=-0.29, \mathrm{p}=0.2)$.

No correlation was found between DTPA clearance and PFTs $\left[\mathrm{FEV}_{1}(\mathrm{r}=-0.016, \mathrm{p}=0.9)\right.$, FVC $(r=0.016, p=0.9)$, TLC $(r=0.14, p=0.6)$ and TLCO $(r=0.2, p=0.5]$, as well as between DTPA clearance or HRCT score and serum angiotensin converting enzyme (SACE).

\section{Discussion}

The present study systematically evaluates newly diagnosed sarcoidosis patients, comparing quantitatively the abnormalities in the HRCT and

Table 1. - Sarcoidosis patients characteristics

\begin{tabular}{lc}
\hline Sex [Male/Female] & $5 / 10$ \\
Age, range, $y r$ & $27-67$ \\
[mean \pm SD] median & {$[47.4 \pm 13.11] 46$} \\
FVC $\%$ pred, $x \pm$ SD & $102.3 \pm 19.75$ \\
FEV $_{1} \%$ pred, \pm \pm SD & $97.9 \pm 21.7$ \\
TLC\% pred, \pm SD & $107.7 \pm 12.3$ \\
TLCO $\%$ pred, $\mathrm{x} \pm \mathrm{SD}$ & $107.9 \pm 14.6$ \\
\hline
\end{tabular}




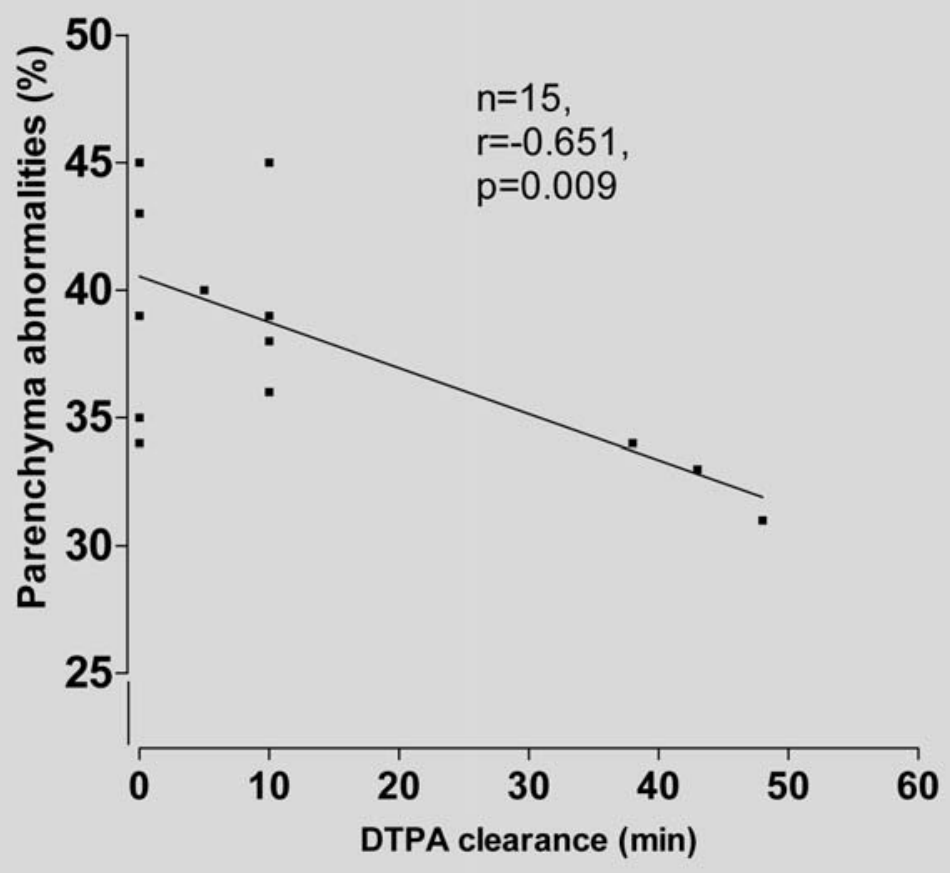

Fig. 1. - Correlation of DTPA clearance and Parenchymal Abnormalities in Sarcoidosis patients.

99mTc-DTPA clearance prospectively. We found that the $99 \mathrm{mTc}-\mathrm{DTPA}$ clearance is abnormal in 10 of 15 of our patients [66.6\%]. The most important finding of our study is a moderate correlation between HRCT parenchymal abnormalities and 99mTc-DTPA clearance time. We also found that HRCT parenchymal abnormalities are significantly correlated with TLCO $[(\mathrm{r}=-0.767)$ and with FVC $(r=-0.65)]$. To the best of our knowledge this is the first study to show the degree of correlation of 99mTc-DTPA clearance and HRCT in sarcoidosis patients.

In this study, all ten sarcoidosis patients with abnormal DTPA scan presented with pulmonary parenchymal involvement on HRCT [type II or III disease] in agreement with previous studies [16, 17]. Furthermore, no correlation existed between 99mTc-DTPA clearance time and PFTs or inflammatory markers like ACE in the serum. In accordance with other studies it seems that DTPA clearance reflects lung function abnormalities better than other tests [16-18].

In addition, we found a correlation between DTPA clearance and HRCT score, although the minimal lung parenchymal lesions in HRCT (12\%). The percentage of HRCT parenchymal abnormalities found in the present study was lower compared to the group of Leung and coworkers reported one to four HRCT features in the total evaluation [19]. Moreover, comparison of HRCT with indices of disease activity (67 Gallium scan, bronchoalveolar lavage [BAL] and SACE) showed that the extent of nodules, and consolidation was well correlated with disease activity. However, activity indices were not correlated with ground glass and linear opacities [19].
Interstitial fibrosis causing functional impairment may develop in up to $20-25 \%$ of patients with sarcoidosis and results in death in 5-10\% [20]. HRCT has been accepted as a non invasive tool for early recognition of interstitial lung involvement in sarcoidosis [21]. Additionally, the extent of the interstitial changes in HRCT was well correlated with respiratory functional impairment [19], while other several studies have reported that HRCT features of air trapping correlated well with obstruction indices in PFTs [22-25]. The nature of the interstitial and airway involvement in sarcoidosis could explain the discrepancies reported in the fore-mentioned studies [21-25].

HRCT parenchymal abnormalities in this study showed a significant correlation with PFTs [TLCO $(r=-0.767)$ and FVC $(r=-0.65)]$. Many research groups have investigated CT as a non invasive examination in distinguishing patients with active disease and potentially reversible lesions from those with irreversible fibrotic disease [26$28]$. Results of these studies though seem to be inconsistent [19, 16, 28-30]. However, two studies Oberstein et al. [12] and Leung et al. [19] reported solid evidence of HRCT correlation with disease activity markers such as BAL total cell and lymphocyte counts, IL-2R, CD4/CD8 ratio, IL-2R and HLA-DR. Their results showed that specific HRCT abnormalities including bronchovascular bundle thickening and intraparenchymal nodules correlate well with the above mentioned disease activity markers [12]. Our scoring system practically similar of that used in the study of Oberstein et al. [12]. This scoring system was recently further evaluated by Drent et al. [31] in 80 consecutive sarcoidosis patients and found significant correlation between 
HRCT scoring and functional parameters. In accordance with these studies the correlation found in this study sustains in all HRCT subscores, except enlargement of lymph nodes.

Mimori Y et al. examined the correlation between the levels of SACE and HRCT in 25 sarcoidosis patients [30]. In this study, the authors did not find a correlation between visual score in HRCT and serum ACE, in according to our data.

Our data suggest that DTPA indicates active disease even in patients with minimal (mild bronchial thickening) or no evidence of HRCT involvement of the lung. However, further studies in a larger number of patients are needed to confirm this correlation of DTPA with the HRCT abnormalities in newly diagnosed sarcoidosis, such as in the follow up of this disease.

\section{References}

1. O'Brodovich H, Coates G. Pulmonary clearance of $99 \mathrm{~m}$ Tc-DTPA: a non invasive assessment of epithelial integrity. Lung 1987; 166: 1-16.

2. Pantin CF, Valind SO, Sweatman M, et al. Measures of the inflammatory response in cryptogenic fibrosing alveolitis. Am Rev Respir Dis 1988; 138: 1234-41.

3. Susskind H. Technetium-99m-DTPA aerosol to measure alveolar-capillary membrane permeability. $\mathrm{J} \mathrm{Nucl}$ Med 1994; 35: 207-9.

4. Chinet T, Dusser D, Labrune S, Collignon MA, Chretien J, Huchon GJ. Lung function declines in patients with pulmonary sarcoidosis and increased respiratory epithelial permeability to 99mTc-DTPA. Am Rev Respir Dis 1990; 141: 445-449.

5. Wells AU, Hansell DM, Harrison NK, Lawrence R, Black CM, du Bois RM. Clearance of inhaled 99mTcDTPA predicts the clinical course of fibrosing alveolitis. Eur Respir J 1993; 6: 797-802.

6. Antoniou KM, Malagari K, Tzanakis N, et al. Clearance of technetium-99m-DTPA and HRCT findings in the evaluation of patients with Idiopathic Pulmonary Fibrosis. BMC Pulm Med 2006 Feb 16; 6: 4.

7. Smith RJ, Hyde RW, Waldman DL, et al. Effect of pattern of aerosol inhalation on clearance of technetium99m-labeled diethylenetriamine pentaacetic acid from the lungs of normal humans. Am Rev Respir Dis 1992; 145: 1109-1116.

8. Smith RJ, Hyde RW, Waldman DL, et al. Radioaerosol clearance in patients with active pulmonary sarcoidosis. Am Rev Respir Dis 1985; 131: 687-689.

9. Raghu G. Interstitial lung disease: a diagnostic approach: are CT scan and lung biopsy indicated in every patient? Am J Respir Crit Care Med 1995; 151: 909-914.

10. Costabel U, Hunninghake GW. ATS/ERS/WASOG statement on sarcoidosis. Sarcoidosis Statement Committee. American Thoracic Society/European Respiratory Society/ World Association of Sarcoidosis and Granulomatous Disorders. Eur Respir J 1999; 14: 735-71.

11. American Thoracic Society. Standardization of spirometry, 1994 update. Am J Respir Crit Care Med 1995; 152: 1107-1136.

12. Oberstein A, von Zitzewitz H, Schweden F, Muller-Quernheim J. Non invasive evaluation of the inflammatory activity in sarcoidosis with high-resolution computed tomography. Sarcoidosis Vasc Diffuse Lung Dis 1997; 14: 65-72.

13. Wells A. High resolution computed tomography in sarcoidosis: a clinical perspective. Sarcoidosis Vasc Diffuse Lung Dis 1998; 15: 140-146.
14. Mogulkoc N, Brutsche MH, Bishop PW, et al. Manchester Pulmonary Fibrosis Consortium. Pulmonary 99mTc-DTPA aerosol clearance and survival in usual interstitial pneumonia [UIP]. Thorax 2001; 56: 916-923.

15. Kon OM, Daniil Z, Black CM, du Bois RM. Clearance of inhaled technetium-99m-DTPA as a clinical index of pulmonary vascular disease in systemic sclerosis. Eur Respir J 1999; 13: 133-136.

16. Dusser DJ, Collignon MA, Stanislas-Leguern G, Barritault LG, Chretien J, Huchon GJ. Respiratory clearance of $99 \mathrm{mTc}-D T P A$ and pulmonary involvement in sarcoidosis. Am Rev Respir Dis 1986; 134: 493-7.

17. Bradvik I, Wollmer P, Evander E, et al. One year follow-up of lung clearance of $99 \mathrm{mTc}$ - dirthylene triamine penta-acetic acid and disease activity in sarcoidosis. Sarcoidosis Vasc Diffuse Lung Dis 2000; 17: 281-287.

18. Watanabe N, Inoue T, Oriuchi N, Suzuki H, Hirano T, Endo K. Increased pulmonary clearance of aerosolised 99Tcm-DTPA in patients with a subset of stage I sarcoidosis. Nucl Med Commun 1995; 16: 464-467.

19. Leung AN, Brauner MW, Caillat-Vigneron N, Valeyre D, Grenier P. Sarcoidosis activity: correlation of HRCT findings with those of $67 \mathrm{Ga}$ scanning, bronchoalveolar lavage, and serum angiotensin-converting enzyme assay. J Comput Assist Tomogr 1998; 22: 229-234.

20. Crystal RG, Roberts RC, Hunninghake GW, et al. Pulmonary sarcoidosis: a disease characterized and perpetuated by activated lung T-lymphocytes. Ann Intern Med 1981; 94: 73-94.

21. Hunninghake GW, Crystal RG. Pulmonary sarcoidosis: a disorder mediated by excess helper T-lymphocyte activity at sites of disease activity. Pulmonary sarcoidosis: morphologic associations of airflow obstruction at thinsection CT. Radiology 1998; 209: 697-704.

22. Magkanas E, Voloudaki A, Bouros D, et al. Pulmonary sarcoidosis. Correlation of expiratory high-resolution CT findings with inspiratory patterns and pulmonary function tests. Acta Radiol 2001; 42: 494-501.

23. Fazzi P, Sbragia P, Solfanelli S, Troilo S, Giuntini C. Functional significance of the described attenuation sign on expiratory CT in pulmonary sarcoidosis. Chest 2001; 119: 1270-1274.

24. Bartz RR, Stern EJ. Airways Obstruction in patients with sarcoidosis. Expiratory CT Scan Findings. Journal of Thoracic Imaging 2000; 15: 285-289.

25. Davies CW, Tasker AD, Padley SPG, Davies RJO, Gleeson FV. Air trapping in Sarcoidosis on computed tomography: correlation with lung function. Clin Radiol 2000; 55: 217-221.

26. Lynch DA, Webb WR, Gamsu G, Stulbarg M, Golden J. Computed tomography in pulmonary sarcoidosis. $J$ Comput Assist Tomogr 1989; 13: 405-410.

27. Brauner MW, Lenoir S, Grenier P, Cluzel P, Battesti JP, Valeyre D. Pulmonary sarcoidosis: CT assessment of lesion reversibility. Radiology 1992; 182: 349-354.

28. Remy-Jardin M, Giraud F, Remy J, Wattinne L, Wallaert B, Duhamel A Pulmonary sarcoidosis: role of CT in the evaluation of disease activity and functional impairment and in prognosis assessment. Radiology 1994; 191: 675-680.

29. Bergin CJ, Bell DY, Coblentz CL, et al. Sarcoidosis: correlation of pulmonary parenchymal pattern at CT with results of pulmonary function tests. Radiology 1989; 171: 619-624.

30. Mimori Y. Sarcoidosis: correlation of HRCT findings with results of pulmonary function tests and serum angiotensin-converting enzyme assay. Kurume Med $\mathrm{J}$ 1998; 45: 247-256.

31. Drent M, De Vries J, Lenters M, et al. Sarcoidosis: assessment of disease severity using HRCT. Eur Radiol 2003; 13: 2462-71. 